\title{
The LHCb experiment: status and expected physics performance
}

\author{
Sergey Barsuk ${ }^{\mathrm{a}}$ on behalf of the LHCb Collaboration

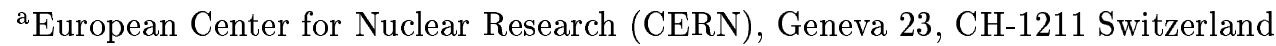 \\ and \\ Institute for Theoretical and Experimental Physics (ITEP), B.Cheremushkinskaya 25, Moscow 117218, \\ Russia
}

Physics motivation of the LHCb experiment, detector design, status of construction and installation of detector components, and expected performance of selected $b$ physics measurements are discussed.

$\mathrm{LHCb}$ is a dedicated experiment at LHC to study CP violation and other rare phenomena in $b$ hadron decays ${ }^{1}$. The awaited contributions from the $\mathrm{LHCb}$ experiment are the precision measurements of the CKM parameters [1] and test the Standard Model (SM) predictions hoping to observe phenomena non-explainable within SM[2].

At the LHC energies, the production of $b$ and $\bar{b}$ quarks are highly correlated with respect to the boost (Fig. 1), so that if one $b$ quark goes into the detector acceptance, the corresponding $\bar{b}$ quark products are also captured with high probability. This proves high efficiency of a single arm spectrometer philosophy, where only a factor 2 is lost compared to a $4 \pi$ geometry. Moreover owing to better acceptance in forward direction, a factor 2 better $b \bar{b}$ cross section is expected at the $\mathrm{LHCb}$ compared to the ATLAS or CMS experiments.

$\mathrm{LHCb}$ will run at a reduced LHC luminosity tuned in the range $2 \div 5 \times 10^{32} \mathrm{~cm}^{-2} \mathrm{~s}^{-1}$ in order to optimize the number of beam crossings with single $p p$ interaction.

The prolific production of the $b$ hadrons at the LHCb is expected with the annual yield of

\footnotetext{
${ }^{1}$ The LHCb reach in $c, \tau$ physics, jets, etc. is beyond the
} scope of this paper. a $10^{12} b \bar{b}$ pairs. All the $b$ species will be produced with the biggest samples of $B_{u}, B_{d}, B_{s}$ and $\Lambda_{b}, B_{u}: B_{d}: B_{s}: B_{c}: \Lambda_{b} \approx 4: 4: 1: 0.1: 1$. While the lightest, $B_{u}$ and $B_{d}$, mesons are being explored in great detail by $B$ factories, the LHCb experiment can contribute to the studies of $B_{s}$ (in particular time resolved studies), $B_{c}$ and $\Lambda_{b}$. Also numerous excited states will be produced allowing the systematic study of the $b$ hadron spectroscopy. There the LHCb will mainly be competing with Super $B$-factory and low luminosity runs of the ATLAS and CMS experiments.

At $\mathrm{LHCb}$ the cross section of $b \bar{b}$ production amounts to less than $1 \%$ of the total cross section. The first level trigger aims at reducing background $\sim 40$ times, so that for high level trigger dominant background originates from charm and beauty events. The goal is to exclusively reconstruct the $B$ meson decay of interest, measure proper time even for fast oscillating $B_{s}$ mesons and provide reliable tagging of the $b$ flavour. Thus the crucial features of the experiment are robust and efficient trigger, particle identification and decay time resolution. Also important particularly for asymmetry measurements is to develop tools for control of the systematic uncer- 


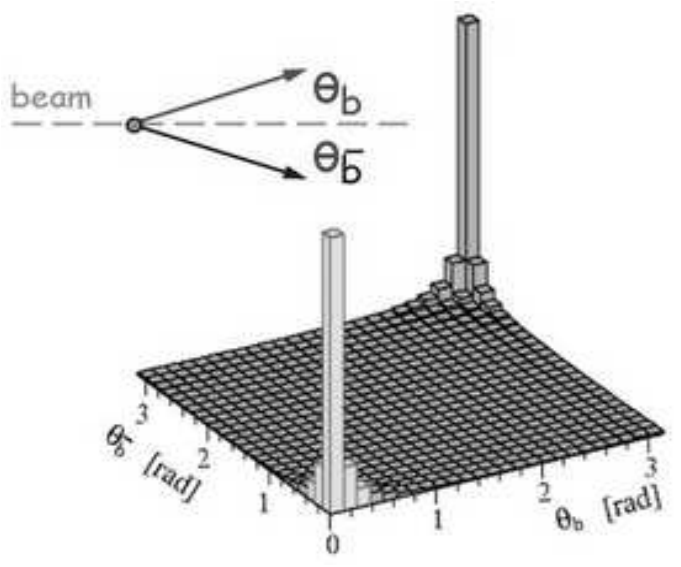

Figure 1. Polar angle distribution of the $b$ and $\bar{b}$ hadrons produced at LHC

tainties [4].

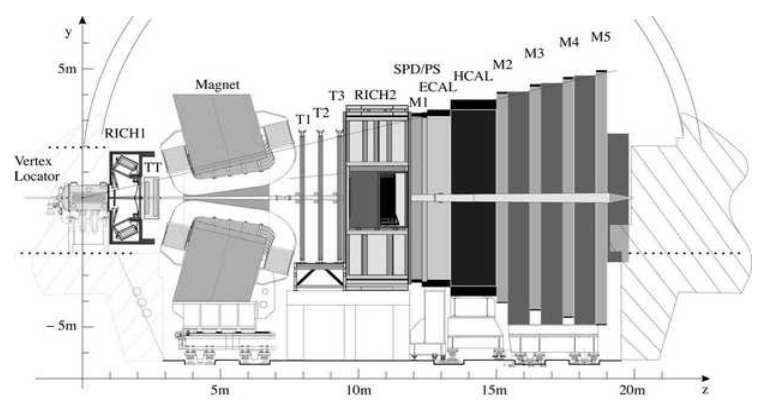

Figure 2. Layout of the $\mathrm{LHCb}$ detector

The layout of $\mathrm{LHCb}$ detector $[5,6]$ is shown on Fig. 2.

Charged particle tracks and vetices will be reconstructed using silicon stations of VErtex LOcator (VELO), Trigger Tracker (TT) station upstream the magnet, and the three tracking stations T1-T3 downstream the magnet [7]. Photons and $\pi^{o}$ mesons will be measured in the electromagnetic calorimeter.

The $K / \pi$ separation is done using the two Cherenkov detectors, RICH1 upstream the magnet and RICH2 downstream the magnet [8]. For $\gamma / e / h$ separation used is the calorimeter consisting of scintillating pad detector (SPD), preshower detector (PS), electromagnetic (ECAL) and hadronic (HCAL) calorimeters. The five muon stations M1-M5 interlayed with the muon filters are used for muon identification.

VELO is a silicon microstrip detector of a $180 \mathrm{k}$ readout channels, installed inside the vacuum vessel (Fig. 3) around the interaction region [9]. The

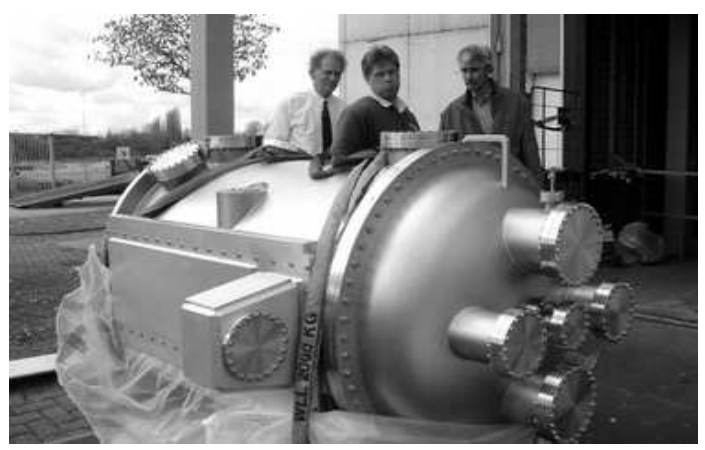

Figure 3. Vacuum vessel for VELO arrived at NIKHEF in $04 / 2005$

detector comprises 21 stations, each with $r$ and $\phi$ measuring sensors, with the silicon $300 \mu$ thick and the pitch varying from 40 to $100 \mu$ depending on radius (Fig. 4, left). VELO is arranged in two retractable detector halves, where the sensors are as close as $8 \mathrm{~mm}$ to the beam line in operation mode, and are $30 \mathrm{~mm}$ away during injection. For primary vertex reconstruction the detector will provide resolution of $44 \mu$ along the beam line and $8 \mu$ in transverse direction. The impact parameter will be determined to a $30 \mu$ precision. For each VELO half, pattern recognition and alignment will be pre-adjusted using the test beam in 2006 before installation in the $\mathrm{LHCb}$. 

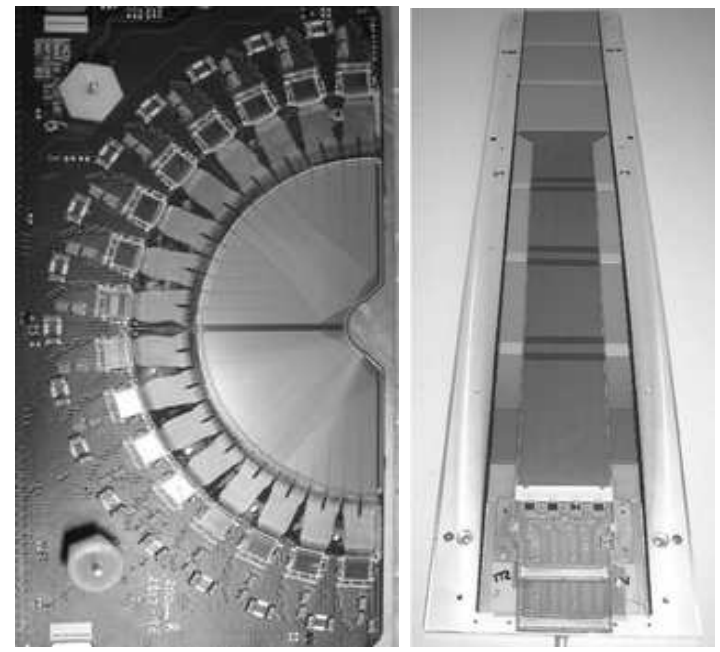

Figure 4. Left: the VELO hybrid with silicon sensor and readout chips; right: sensor ladder for TT and IT with electronics hybrid, low mass Kapton cable and silicon sensors

The warm dipole magnet [10] provides an integrated field of $4 \mathrm{Tm}$. The magnetic field will be regularly reversed to reduce systematic error in $\mathrm{CP}$ studies. Construction of the magnet is com-

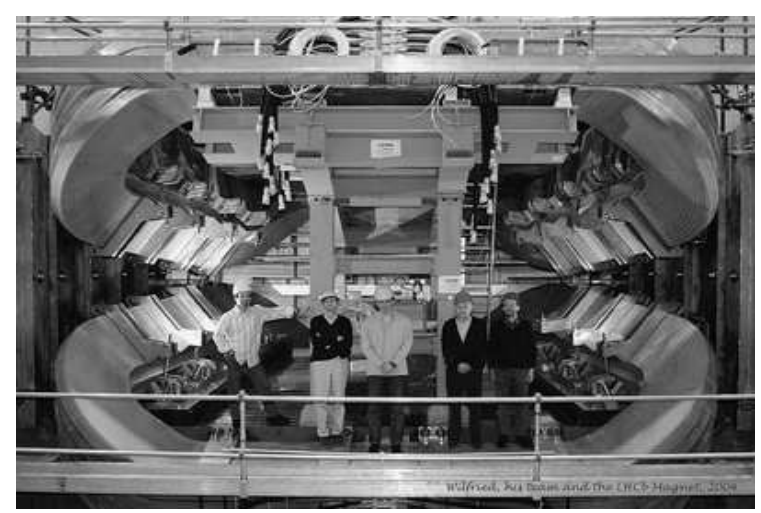

Figure 5. The LHCb magnet pleted (Fig. 5), and the field mapping is ongoing.

TT is a silicon strip detector (Fig. 4, right) covering full acceptance upstream the magnet, with the total $8 \mathrm{~m}^{2}$ of $500 \mu$ thick silicon, $144 \mathrm{k}$ readout channels [11]. T1-T3 stations also exploit silicon strips for inner part (IT) having $129 \mathrm{k}$ readout channels [11], and long straw tube chambers technology for the outer part (OT) having $54 \mathrm{k}$ readout channels [12]. The production of the silicon tracker is being started, while about $60 \%$ of OT chambers is already produced and tested. The tracker gives $\sim 0.4 \%$ momentum resolution with efficiency of $\sim 94 \%$ for fast $(p>10 \mathrm{GeV} / \mathrm{c})$ tracks and ghost rate of $\sim 3 \%$ for tracks with $p_{T}>0.5 \mathrm{GeV} / \mathrm{c}$.

In order to provide good $K / \pi$ separation for momentum range from 2 to $100 \mathrm{GeV} / \mathrm{c}$ the system of Cherenkov detectors [13] uses three radiators: silica aerogel, $C_{4} F_{10}$ in RICH1 to identify low momentum tracks over full acceptance and $C F_{4}$ gas in RICH2 for high momentum tracks. The RICH1 vessel in under construction, and more than 50\% of high quality aerogel is already produced. The shielding for photodetectors is installed in the LHCb (Fig. 6, left). The RICH2 vessel is com-
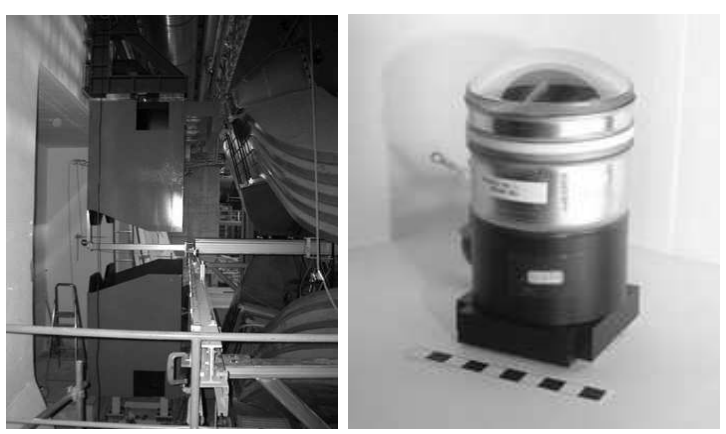

Figure 6. Left: shielding for RICH1 photodetectors installed in $\mathrm{LHCb}$; right: hybrid photodiod

pleted, the mirrors installed and aligned. Both detectors use a dedicated photon detector, hy- 
brid photodiod (HPD), $8 \mathrm{~cm}$ diameter tube, with over 1000 pixels sensitive to single photoelectrons (Fig. 6, right). The production of a $500 \mathrm{HPDs}$ is ongoing.

The CALO system [14] consists of SPD/PS structure (two $15 \mathrm{~mm}$ scintillator layers with 14 $m m$ lead inbetween), ECAL and HCAL, put in projective geometry, and having variable granularity. SPD/PS detector (Fig. 7) employes deep groove design of the cells, $2 \times 6 \mathrm{k}$ cells in total, with the light read out with the 64 channel multianode photomultipliers. The produc-

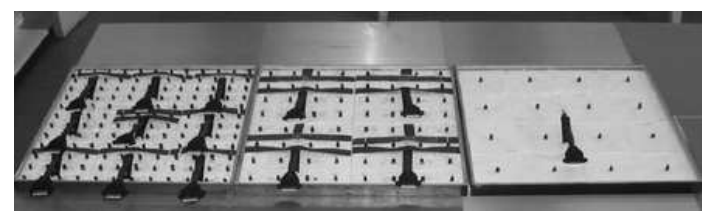

Figure 7. The SPD/PS modules with 144, 64 and 16 detector cells

tion of SPD/PS is ongoing. The ECAL modules employs Shashlyk technology with volume ratio $P b: S c=2: 4(\mathrm{~mm})$, with $6 \mathrm{k}$ readout cells, having $25 X_{o}$ depth and transverse granularity of 4,6 and $12 \mathrm{~cm}$ cell size (Fig. 8, up). The detector production is completed, all the modules are installed in the LHCb. The HCAL is iron-scintillator tile calorimeter, $5.6 \lambda$ deep, having $1.5 \mathrm{k}$ readout cells (Fig. 8, down). The detector production is completed, all the modules are installed in the $\mathrm{LHCb}$.

Muon detector stations are composed of $1.4 \mathrm{k}$ microwire proportional chambers with projective pad readout [15]. A $30 \%$ of chambers are produced and tested. Muon system provides muon identification with a $95 \%$ efficiency with a fake rate of less than $1 \%$.

LHCb trigger [16,17] fully exploits $b$ decay topology, where substantial energy is released yielding high values of $p_{T}$, and $b$ decay vertex is separated from its production vertex by $\sim 1 \mathrm{~cm}$ yielding large impact parameter (IP) values. The

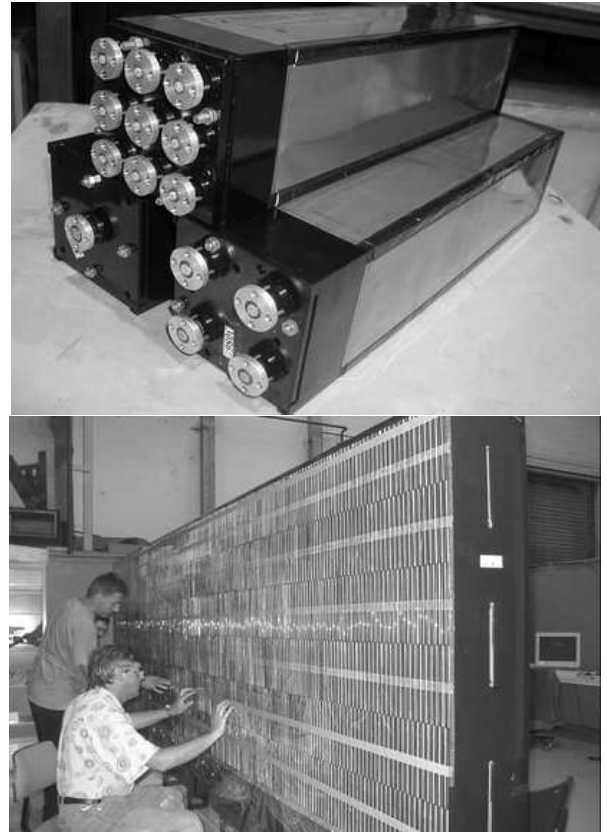

Figure 8. Up: ECAL modules with different cell size; down: optics assembly of HCAL module

level 0 hardware trigger relies on high- $p_{T} e, \gamma, \pi^{o}$, $\mu$ or $h$ candidates, while removing busy events ${ }^{2}$, and decreases the event rate from 40 to $1 \mathrm{MHz}$. All higher level triggers are software and based on the same farm, they add first the IP discrimination, and then the information from the full detector.

Selected baseline physics measurements are discussed below. The related simulation [3] was based on the full detector simulation with pile-up and spill-over included, realistic digitization and reconstruction with full pattern recognition and realistic trigger simulation, with tuned toy Monte Carlo for sensitivity studies. The background was assumed to come from $b \bar{b}$ inclusive events, having similar topology.

To measure $B_{s} \bar{B}_{s}$ oscillations LHCb will fully

\footnotetext{
${ }^{2}$ In order to veto busy events, the information from pile-up system of VELO and the total transverse energy deposited in the HCAL will be used.
} 
reconstruct the decay $B_{s} \rightarrow D_{s} \pi$. Having the proper time resolution of $\sim 40 \mathrm{fs}$, a $80 \mathrm{k}$ events will be observed per 1 year of running ${ }^{3}$. A $5 \sigma$ observation of mixing is possible up to $\Delta m_{s}$ of $68 \mathrm{ps}^{-1}$ which is well beyond the SM expectation. Once the effect is seen, the oscillation frequency is precisely determined with $\sigma_{\text {stat }}\left(\Delta m_{s}\right) \sim 0.01 \mathrm{ps}^{-1}$.

The angle $\gamma$ is feasible at $\mathrm{LHCb}$ in three different approaches. The most clean information should come from the measurement of $\gamma-2 \chi$ from the $B_{s} \rightarrow D_{s} K$ decay, where only tree diagrams contribute and no new physics effects are expected, potential contribution to the $B_{s}$ mixing phase $\phi_{s}=-2 \chi$ will be deducted from the independent measurement (see below). Here $K / \pi$ separation allows to significantly suppress dangerous reflection from $B_{s} \rightarrow D_{s} \pi$. A $5.4 \mathrm{k}$ events annual sample is expected with $^{4}$ $B / S<1$. For $\Delta m_{s}=20 p s^{-1}$ this measurement gives $\sigma(\gamma)$ of $14^{\circ}$. Another way of measuring $\gamma$ comes from time-dependent asymmetries for $B_{d} \rightarrow \pi \pi$ and $B_{s} \rightarrow K K, A_{C P}(t)=$ $A^{d i r} \cos (\Delta m t)+A^{m i x} \sin (\Delta m t)$. Four observables are parameterized with seven parameters, $\gamma, \phi_{d}$ and $\phi_{s}$, and ratios of penguin over tree contribution, $P / T=d e^{i \theta}$. Taking the $\phi_{d}$ and $\phi_{s}$ from other measurements and assuming $U$-spin symmetry [18], we arrive at 4 measurements with 3 unknowns, which we solve for $\gamma$. Here again $K / \pi$ separation is crucial because of numerous reflections from $B_{d, s}$ and $\Lambda_{b}$ decays. The annual yield of $25 \mathrm{k}$ events for $B_{d} \rightarrow \pi \pi$ and $37 \mathrm{k}$ for $B_{s} \rightarrow K K$ gives $\sigma(\gamma)$ of $5^{\circ}$. This measurement is sensitive to new physics in penguin loops. Having the system overconstrained, uncertainty from $U$-spin assumption can be evaluated. Another method to determine $\gamma$ comes from analysis of $B_{d} \rightarrow D K^{*}$ decays [19], which is sensitive to new physics in $D \bar{D}$ mixing.

The $B_{s} \rightarrow J / \Psi \phi, B_{s} \rightarrow J / \Psi \eta$ and $B_{s} \rightarrow \eta_{c} \phi$ decays measure $\phi_{s}=-2 \chi$ and $\Delta \Gamma_{s} / \Gamma_{s}$ simultaneously $^{5}$. $B_{s} \rightarrow J / \Psi \phi$ provides more statistics, but requires partial wave analysis, being a non-CP

\footnotetext{
${ }^{3}$ One year of LHCb running corresponds to $2 \mathrm{fb}^{-1}$ accumulated statistics.

${ }^{4}$ The $90 \%$ CL is assumed throughout the paper.

${ }^{5}$ The SM values are -0.04 and 0.1 correspondingly.
}

state (Table 1 ). The 1 year LHCb combined $\phi_{s}$

Table 1

Sensitivity on $\Delta \Gamma_{s}$ and $\phi_{s}$ for $1 \mathrm{LHCb}$ year

\begin{tabular}{lrccl}
\hline & $\begin{array}{r}\text { annual } \\
\text { yield }\end{array}$ & $\mathrm{B} / \mathrm{S}$ & $\sigma\left(\Delta \Gamma_{s} / \Gamma_{s}\right)$ & $\begin{array}{l}\sigma\left(\phi_{s}\right) \\
{[\mathrm{rad}]}\end{array}$ \\
\hline$B_{s} \rightarrow J / \Psi \phi$ & $100 \mathrm{k}$ & $<0.3$ & 0.018 & 0.06 \\
$B_{s} \rightarrow J / \Psi \eta$ & $7 \mathrm{k}$ & $<5.1$ & 0.025 & 0.1 \\
$B_{s} \rightarrow \eta_{c} \phi$ & $3.2 \mathrm{k}$ & $<1.4$ & 0.025 & 0.1 \\
combined & & & & 0.05 \\
\hline
\end{tabular}

sensitivity of $\sim 0.05$ is comparable to the predicted value, 5 years sensitivity is $\sigma\left(\phi_{s}\right) \sim 0.02$.

Rare loop-induced decays are sensitive to new physics in many SM extentions. At LHCb studies of radiative penguin decays $B_{d} \rightarrow K^{*} \gamma, B_{s} \rightarrow \phi \gamma$, $B_{d} \rightarrow \omega \gamma$, electroweak penguin decay $B_{d} \rightarrow K^{*} \mu \mu$, gluonic penguin decays $B_{d} \rightarrow \phi K_{s}, B_{s} \rightarrow \phi \phi$ and the decay $B_{s} \rightarrow \mu \mu$ will be performed [20]. The expected annual event yields and background over signal levels for rare decays are summarized in Table 2.

Table 2

Expected annual event yields and background over signal levels for rare decays

\begin{tabular}{lcc}
\hline channel & annual yield & $B / S$ \\
\hline$B_{d} \rightarrow K^{*} \gamma$ & $35 \mathrm{k}$ & $<0.7$ \\
$B_{s} \rightarrow \phi \gamma$ & $9.3 \mathrm{k}$ & $<2.4$ \\
$B_{d} \rightarrow \omega \gamma$ & $0.04 \mathrm{k}$ & $<3.5$ \\
$B_{d} \rightarrow K^{*} \mu \mu$ & $4.4 \mathrm{k}$ & $<2.0$ \\
$B_{d} \rightarrow \phi K_{s}$ & $0.8 \mathrm{k}$ & $<0.2$ \\
$B_{s} \rightarrow \phi \phi$ & $1.2 \mathrm{k}$ & $<1.1$ \\
$B_{s} \rightarrow \mu \mu$ & $0.02 \mathrm{k}$ & $<440$ \\
\hline
\end{tabular}

The decay $B_{c} \rightarrow J / \Psi \pi$ illustrates the $\mathrm{LHCb}$ reach of heavier $B$ mesons. The expected yield is $14 \mathrm{k}$ events per year of running with $B / S<0.8$.

Selected performance results are summarized in Table 3 . 
Table 3

Selected performance after $1 \mathrm{LHCb}$ year

\begin{tabular}{llll}
\hline & channel & yield & precision \\
\hline$\gamma$ & $B_{s} \rightarrow D_{s} K$ & $5.4 \mathrm{k}$ & $\sigma(\gamma) \approx 14^{o}$ \\
\cline { 2 - 4 } & $B_{d} \rightarrow \pi \pi$ & $26 \mathrm{k}$ & $\sigma(\gamma) \approx 6^{\circ}$ \\
& $B_{s} \rightarrow K K$ & $37 \mathrm{k}$ & \\
\cline { 2 - 4 } & $B_{d} \rightarrow D^{o} K^{*}$ & $0.5 \mathrm{k}$ & \\
& $B_{d} \rightarrow \bar{D}^{o} K^{*}$ & $3.4 \mathrm{k}$ & $\sigma(\gamma) \approx 8^{o}$ \\
& $B_{d} \rightarrow D_{C P} K^{*}$ & $0.6 \mathrm{k}$ & \\
\hline$\chi$ & $B_{s} \rightarrow J / \Psi \phi$ & $120 \mathrm{k}$ & $\sigma(\chi) \approx 2^{o}$ \\
\hline$\left|V_{t d} / V_{t s}\right|$ & $B_{s} \rightarrow D_{s} \pi$ & $80 \mathrm{k}$ & $\Delta m_{s}$ up \\
& & & to $68 p s^{-1}$ \\
\hline$A_{C P}^{\text {dir }}$ & $B_{d} \rightarrow K^{*} \gamma$ & $35 \mathrm{k}$ & $\sigma\left(A_{C P}^{\text {dir }}\right)$ \\
& & & $\approx 0.01$
\end{tabular}

Statistical errors only are taken into account.

In summary, the $\mathrm{LHCb}$ detector is optimized to exploit huge sample of $10^{12} b$ hadrons per year, including all $b$ species, serving e.g. as a first $B_{(s, c)}$ factory. The physics programme is complementary/competitive to (Super)- $B_{(d, u)}$ factories and Tevatron. The $\mathrm{LHCb}$ construction and installation are well ongoing, the experiment will be installed and commissioned by the LHC start-up in 2007 . The $\mathrm{LHCb}$ physics potential can be fully exploited already with the initial LHC luminosity.

Acknoledgements. The author wishes to thank I. Belyaev, H. Dijksta, D. Eckstein, A. Golutvin, R. Forty, R. Lindner, T. Ruf, O. Schneider, A. Schopper, F. Teubert and O. Ullaland for their help in preparing the talk.

\section{REFERENCES}

1. L. Silvestrini, talk at this conference.

2. T. Hurth, talk at this conference.

3. P. Koppenburg, talk at this conference.

4. G. Wilkinson, talk at this conference.

5. LHCb Technical Proposal, CERN/LHCC 984.

6. LHCb Reoptimized Detector Design and Performance TDR, CERN LHCC 2003-30.

7. M. Needham, talk at this conference.
8. T. Bellunato, talk at this conference.

9. LHCb VELO Technical Design Report, CERN LHCC 2001-011.

10. LHCb Magnet Technical Design Report, CERN LHCC 2000-007.

11. LHCb Inner Tracker Technical Design Report, CERN LHCC 2002-029.

12. LHCb Outer Tracker Technical Design Report, CERN LHCC 2001-024.

13. LHCb RICH Technical Design Report, CERN LHCC 2000-037.

14. LHCb Calorimeter Technical Design Report, CERN LHCC 2000-036.

15. LHCb Muon System Technical Design Report, CERN LHCC 2001-010.

16. LHCb Trigger Technical Design Report, CERN LHCC 2003-031.

17. F. Teubert, talk at this conference.

18. R. Fleischer, Phys.Lett. B 459 (1999) 306.

19. M. Gronau and D. Wyler, Phys.Lett. B 265 (1991) 172;

I. Dunietz, Phys.Lett. B 270 (1991) 75.

20. I. Belyaev, Chech.J.Phys.55 Suppl.A (2005). 